\title{
A Two-Stage Mutation Stochastic Model of Carcinogenesis Driven by a Two Level Random Environment
}

\author{
V.S. S. Yadavalli \\ Department of Industrial and System Engineering, \\ University of Pretoria, Pretoria 0002, South Africa. \\ Corresponding author: Sarma.Yadavalli@up.ac.za \\ S. Udayabaskaran \\ Department of Mathematics, \\ Vel Tech Rangarajan Dr. Sagunthala R\&D Institute of Science and Technology, \\ Avadi, Chennai 600062, India. \\ E-mail: sudayabaskaran@veltech.edu.in \\ C. T. Dora Pravina \\ Department of Mathematics, \\ Vel Tech Rangarajan Dr. Sagunthala R\&D Institute of Science and Technology, \\ Avadi, Chennai 600062, India. \\ E-mail: tdorapravinac@ veltech.edu.in \\ S. Sreelakshmi \\ Department of Engineering Mathematics, \\ HKBK College of Engineering, Bangalore 560045, India. \\ E-mail: sreelakshmi.mt@hkbk.edu.in
}

(Received May 7, 2020; Accepted June 24, 2020)

\begin{abstract}
In this paper, we present a two-stage stochastic model of carcinogenesis in a two level random environment. The random environment switches between two levels, say, 1 and 2 alternately. When the environment is in level 1, a normal cell either divides into two normal cells or dies; and an intermediate cell divides into two intermediate cells or dies. When the environment is in level 2, a normal cell either divides into two intermediate cells or divides into one normal cell and one intermediate cell or divides into two normal cells or dies; and an intermediate cell either divides into two malignant cells or divides into one intermediate cell and one malignant cell or divides into two intermediate cells or dies. It is assumed that, once a malignant cell is produced, it generates a malignant tumor with probability 1 . We obtain the mean numbers of normal, intermediate and malignant cells.
\end{abstract}

Keywords- Age-dependent two-stage stochastic model, Normal cell, Intermediate cell, Malignant cell, Random environment.

\section{Introduction}

Branching processes have been very extensively studied in the past to understand several physical and biological processes (see, for example, Harris, 1963; Srinivasan, 1969; Mode, 1971; Athreya and Ney, 1972; Assmussen and Hering, 1983). Branching character is inherent in cell division processes where in each cell (also called an individual or a particle) lives for some random amount of time and then dies, differentiates or splits into two daughter cells. Sometimes, before a normal cell splits, it undergoes genetic changes (mutational changes) and then splits into daughter cells abnormally different from the characters of their mother. Innumerable genetic changes can take 
International Journal of Mathematical, Engineering and Management Sciences

Vol. 5, No. 6, 1392-1408, 2020

https://doi.org/10.33889/IJMEMS.2020.5.6.103

place during cell division and these mutational transformations lead to cancer cells which are quite different from normal cells. Usually, it is assumed that all particles behave independently and identically; and all particles have a common exponential life-time and a common off-spring probability generating function. Sun et al. (2014) have observed that tumorigenesis can be regarded as an evolutionary process. They have formulated a new model of time scheme for progression of colorectal cancer based upon maturity and predicted the values of several important parameters in cancer progression. Tomasetti and Vogelstein (2015) have postulated that cancer risk is due to random mutations arising during DNA replication in normal, noncancerous stem cells. Rozhok and DeGregori (2019) have presented a theoretical study on the evolution of lifespan and age-dependent cancer risk. Rozhok et al. (2016) have applied a stochastic Monte Carlo model to explain the age-dependent incidence of cancer. Reddy et al. (2017) have observed that during every cell division, some rare events at the genome level such as DNA replication mistakes take place of which some are of no importance, while some have significance for escaping cell division control mechanisms. Martincorena et al. (2017) have concluded that cancer risk is dependent on the random errors occurring in normal cell replication, hereditary defects in critical genes, and environmental factors including exogenous agents and lifestyle. Hochberg and Noble (2017) have provided a framework for understanding how natural environmental variation and human activity impact cancer risk, with potential implications for species ecology. Simulation studies have been reported in the paper of Rozhok and DeGregori (2019) on a generalized theory of age-dependent carcinogenesis to demonstarte the impact of key somatic evolutionary parameters on the performance of Multistage Model of Carcinogenesis. Wolf et al. (2019) have presented an unified theory of carcinogenesis in which they have considered multi-stage carcinogenesis models to assess the carcinogenicity of chemicals for risk management and the public communication. In the above studies, cell division mechanism have either dependance on random mutations arising during DNA replication in normal cells or on environment or external agents such as chemical substances or living habits. This type of mutation-dependent and environment-dependent cell division processes in connection with carcinogenesis has not been studied analytically so far in literature.

The above papers encouraged us to propose and analyze a stochastic model incorporating the growth of the cell population in random medium. As in Moolgavkar and Venzon (1979), we assume that a normal cell generates malignant cells by two mutations. To be specific, in this paper, we present a two-stage stochastic model of carcinogenesis in a two level random environment. The random environment switches between two states 1 and 2 alternately. When the environment is in level 1, a normal cell either divides into two normal cells with rate $L_{11}$ or dies with rate $D_{11}$; and an intermediate cell either divides into two intermediate cells with rate $\alpha_{11}$ or dies with rate $\mu_{11}$. When the environment is in level 2, a normal cell either divides into two intermediate cells with rate $L_{20}$ or divides into one normal cell and one intermediate cell with rate $L_{21}$ or divides into two normal cells with rate $L_{22}$ or dies with rate $D_{22}$; and an intermediate cell either divides into two malignant cells with rate $\alpha_{20}$ or divides into one intermediate cell and one malignant cell with rate $\alpha_{21}$ or divides into two intermediate cells with rate $\alpha_{22}$ or dies with rate $\mu_{22}$. It is assumed that, once a malignant cell is produced, it generates a malignant tumor with probability 1 . We obtain the mean numbers of normal, intermediate and malignant cells.

The rest of this paper is organised as follows. In section 2, we describe the model. In section 3, we write the integral equations satisfied by the conditional probability generating functions of the number of normal, intermediate and malignant cells. In section 4, we derive the mean number of normal, intermediate and malignant cells in the population. We present a numerical illustration in section 5 to highlight the impact of environment on carcinogenesis. We provide a conclusion in 
International Journal of Mathematical, Engineering and Management Sciences

Vol. 5, No. 6, 1392-1408, 2020

https://doi.org/10.33889/IJMEMS.2020.5.6.103

section 6 .

\section{Model Formulation}

We consider a population of cells originating from a single normal cell present at time $t=0$. As time progresses, cell divisions take place and due to mutational changes, malignant cells are produced by a two-stage process. That is, a normal cell first splits into intermediate cells (pre-malignant cells) and then these intermediate cells split into malignant cells. Hence, after a positive time, the cell population will have normal, intermediate and malignant cells. Let $X(t), Y(t)$, and $Z(t)$ be the random variables denoting the number of normal, intermediate, and malignant cells at time $t$ respectively. We define the time duration a cell has lived with out splitting since its birth time as the age of the cell.

\subsection{Assumptions}

(i) All cells evolve in a random environment.

(ii) At any time $t$, the random environment is in one of the two levels, say, 1 and 2.

(iii) Let $\Theta(t)$ be the state of the environment at any time $t$. Then,

$$
\mathbb{P}[\Theta(t+\Delta t)=2 \mid \Theta(t)=1]=\lambda_{12} \Delta t+o(\Delta t),
$$$$
\mathbb{P}[\Theta(t+\Delta t)=1 \mid \Theta(t)=2]=\lambda_{21} \Delta t+o(\Delta t),
$$

$$
\begin{aligned}
& \mathbb{P}[\Theta(t+\Delta t)=1 \mid \Theta(t)=1]=1-\lambda_{12} \Delta t+o(\Delta t), \\
& \mathbb{P}[\Theta(t+\Delta t)=2 \mid \Theta(t)=2]=1-\lambda_{21} \Delta t+o(\Delta t) .
\end{aligned}
$$

(iv) When the environment is in level 1 at time $t$, a normal cell existing at time $t$ either divides into two normal cells with probability $L_{11} \Delta t+o(\Delta t)$ or dies with probability $D_{11} \Delta t+$ $o(\Delta t)$ in the infinitesimal interval $(t, t+\Delta t)$;

(v) When the environment is in level 1 at time $t$, an intermediate cell existing at time $t$ either divides into two intermediate cells with probability $\alpha_{11} \Delta t+o(\Delta t)$ or dies with probability $\mu_{11} \Delta t+o(\Delta t)$ in the infinitesimal interval $(t, t+\Delta t)$;

(vi) When the environment is in state 2 at time $t$, a normal cell existing at time $t$ either divides into two intermediate cells with probability $L_{20} \Delta t+o(\Delta t)$ or divides into one normal cell and one intermediate cell with probability $L_{21} \Delta t+o(\Delta t)$ or divides into two normal cells with probability $L_{22} \Delta t+o(\Delta t)$ or dies with probability $D_{22} \Delta t+o(\Delta t)$ in the infinitesimal interval $(t, t+\Delta t)$;

(vii) When the environment is in state 2 at time $t$, an intermediate cell existing at time $t$ either divides into two malignant cells with probability $\alpha_{20} \Delta t+o(\Delta t)$ or divides into one intermediate cell and one malignant cell with probability $\alpha_{21} \Delta t+o(\Delta t)$ or divides into two intermediate cells with probability $\alpha_{22} \Delta t+o(\Delta t)$ or dies with probability $\mu_{22} \Delta t+$ $o(\Delta t)$ in the infinitesimal interval $(t, t+\Delta t)$.

(viii) Once a malignant cell is produced, it generates a malignant tumor with probability 1.

(ix) All events are independent and the probability of occurrence of more than one event in a small interval $(t, t+\Delta t)$ is $o(\Delta t)$.

\section{Governing Equations}

Assume that we start with 1 newly born normal cell and no other cells. Then, we have the condition 
International Journal of Mathematical, Engineering and Management Sciences

Vol. 5, No. 6, 1392-1408, 2020

https://doi.org/10.33889/IJMEMS.2020.5.6.103

$X(0)=1, Y(0)=0, Z(0)=0$ at time $t=0$. It is clear that the four-dimensional process $\{(X(t), Y(t), Z(t), \Theta(t)): t \geq 0\}$ is Markov. The state-transition diagram is given in Figure 1 below:

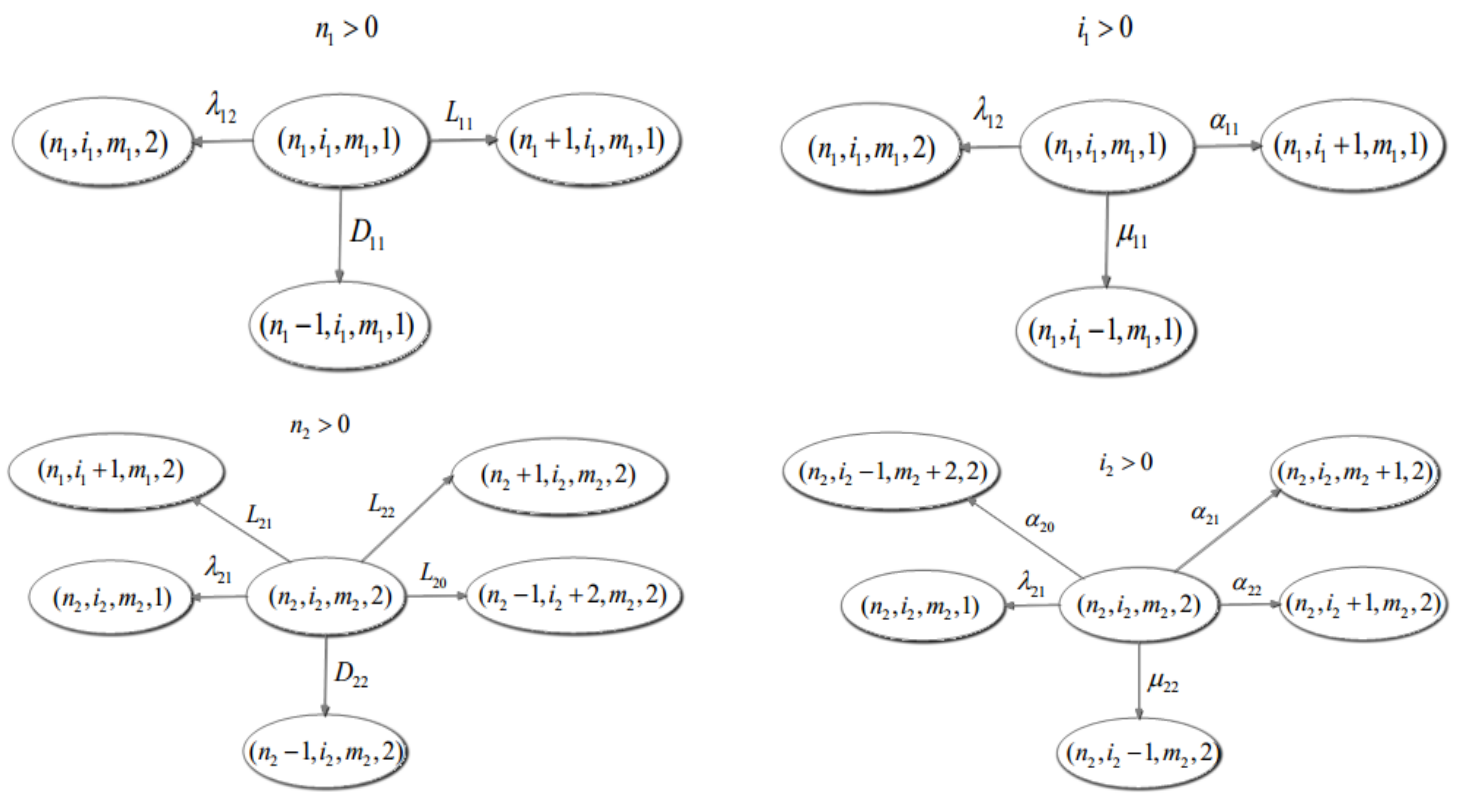

Figure 1. State transition diagram

We define the conditional probability generating functions for the number of normal, intermediate, and malignant cells at time $t$ initiated by a single normal cell at time $t=0$ as follows:

$$
\begin{aligned}
& \psi_{1}(x, y, z, t)=\mathbb{E}\left[x^{X(t)} y^{Y(t)} z^{Z(t)} \mid X(0)=1, Y(0)=0, Z(0)=0, \Theta(0)=1\right] \\
& \psi_{2}(x, y, z, t)=\mathbb{E}\left[x^{X(t)} y^{Y(t)} z^{Z(t)} \mid X(0)=1, Y(0)=0, Z(0)=0, \Theta(0)=2\right]
\end{aligned}
$$

In the same manner, we define the conditional probability generating function for the number of intermediate and malignant cells at time $t$ initiated by a single intermediate cell at time $t=0$ as follows:

$$
\begin{aligned}
& \phi_{1}(y, z, t)=\mathbb{E}\left[y^{Y(t)} Z^{Z(t)} \mid Y(0)=1, Z(0)=0, \Theta(0)=1\right], \\
& \phi_{2}(y, z, t)=\mathbb{E}\left[y^{Y(t)} Z^{Z(t)} \mid Y(0)=1, Z(0)=0, \Theta(0)=2\right] .
\end{aligned}
$$

For notational simplicity, we write $\psi_{n}(x, y, z, t), \phi_{m}(y, z, t)$ as $\psi_{n}(t), n=1,2$ and $\phi_{m}(t), m=$ 1,2 respectively. Considering the first event (if any) happening in the time interval $(0, t)$ and using the invariant imbedding technique (see Bellmann et al.,1960), we obtain integral equations for $\psi_{n}(t)$ and $\phi_{m}(t)$. To get $\psi_{1}(t)$, we note that the following mutually exclusive and exhaustive cases occur: 
International Journal of Mathematical, Engineering and Management Sciences

Vol. 5, No. 6, 1392-1408, 2020

https://doi.org/10.33889/IJMEMS.2020.5.6.103

(i) no event takes place before $t$; that is, the environment does not change its level before $t$ and the normal cell with which we started at time $t=0$ neither splits nor dies before $t$;

(ii) the first event happening in $(0, t)$ is that the environment changes its level from 1 to 2 in the interval $(u, u+\Delta t) \subseteq(0, t)$;

(iii) the first event happening in $(0, t)$ is that the normal cell with which we started at time $t=$ 0 splits into two normal cells in the interval $(u, u+\Delta t) \subseteq(0, t)$;

(iv) the first event happening in $(0, t)$ is that the normal cell with which we started at time $t=$ 0 dies (or differentiate) in the interval $(u, u+\Delta t) \subseteq(0, t)$.

Consequently, we have

$$
\begin{gathered}
\psi_{1}(t)=x e^{-A t}+\lambda_{12} \int_{0}^{t} e^{-A u} \psi_{2}(t-u) d u+L_{11} \int_{0}^{t} e^{-A u}\left\{\psi_{1}(t-u)\right\}^{2} d u \\
+\frac{D_{11}}{A}\left(1-e^{-A t}\right)
\end{gathered}
$$

where, $A=\lambda_{12}+L_{11}+D_{11}$.

To get the equation for $\psi_{2}(t)$, we note that the following mutually exclusive and exhaustive cases occur:

(i) no event takes place before $t$; that is, the environment does not change its level before $t$ and the normal cell with which we started at time $t=0$ neither splits nor dies before $t$;

(ii) the first event happening in $(0, t)$ is that the environment changes its level from 2 to 1 in the interval $(u, u+\Delta t) \subseteq(0, t)$;

(iii) the first event happening in $(0, t)$ is that the normal cell with which we started at time $t=$ 0 splits into two normal cells in the interval $(u, u+\Delta t) \subseteq(0, t)$;

(iv) the first event happening in $(0, t)$ is that the normal cell with which we started at time $t=$ 0 splits into one normal cell and one intermediate cell in the interval $(u, u+\Delta t) \subseteq(0, t)$;

(v) the first event happening in $(0, t)$ is that the normal cell with which we started at time $t=$ 0 splits into two intermediate cells in the interval $(u, u+\Delta t) \subseteq(0, t)$;

(vi) the first event happening in $(0, t)$ is that the normal cell with which we started at time $t=$ 0 dies (or differentiate) in the interval $(u, u+\Delta t) \subseteq(0, t)$.

Consequently, we have

$$
\begin{gathered}
\psi_{2}(t)=x e^{-B t}+\lambda_{21} \int_{0}^{t} e^{-B u} \psi_{1}(t-u) d u+L_{22} \int_{0}^{t} e^{-B u}\left\{\psi_{2}(t-u)\right\}^{2} d u+ \\
L_{21} \int_{0}^{t} e^{-B u} \psi_{2}(t-u) \phi_{2}(t-u) d u+L_{20} \int_{0}^{t} e^{-B u}\left\{\phi_{2}(t-u)\right\}^{2} d u \\
+\frac{D_{22}}{B}\left(1-e^{-B t}\right)
\end{gathered}
$$

where, $B=\lambda_{21}+L_{22}+L_{21}+L_{20}+D_{22}$.

To get the equation for $\phi_{1}(t)$, we note that the following mutually exclusive and exhaustive cases occur: 
International Journal of Mathematical, Engineering and Management Sciences

Vol. 5, No. 6, 1392-1408, 2020

https://doi.org/10.33889/IJMEMS.2020.5.6.103

(i) no event takes place before $t$; that is, the environment does not change its level before $t$ and the intermediate cell with which we started at time $t=0$ neither splits nor dies before $t$;

(ii) the first event happening in $(0, t)$ is that the environment changes its level from 1 to 2 in the interval $(u, u+\Delta t) \subseteq(0, t)$;

(iii) the first event happening in $(0, t)$ is that the intermediate cell with which we started at time

(iv) $t=0$ splits into two intermediate cells in the interval $(u, u+\Delta t) \subseteq(0, t)$;

(v) the first event happening in $(0, t)$ is that the intermediate cell with which we started at time

(vi) $t=0$ dies (or differentiate) in the interval $(u, u+\Delta t) \subseteq(0, t)$.

Consequently, we have

$$
\begin{gathered}
\phi_{1}(t)=y e^{-a t}+\lambda_{12} \int_{0}^{t} e^{-a u} \phi_{2}(t-u) d u+\alpha_{11} \int_{0}^{t} e^{-a u}\left\{\phi_{1}(t-u)\right\}^{2} d u \\
+\frac{\mu_{11}}{a}\left(1-e^{-a t}\right)
\end{gathered}
$$

where, $a=\lambda_{12}+\alpha_{11}+\mu_{11}$.

To get the equation for $\phi_{2}(t)$, we note that the following mutually exclusive and exhaustive cases occur:

(i) no event takes place before $t$; that is, the environment does not change its level before $t$ and the intermediate cell with which we started at time $t=0$ neither splits nor dies before $t$;

(ii) the first event happening in $(0, t)$ is that the environment changes its level from 2 to 1 in the interval $(u, u+\Delta t) \subseteq(0, t)$;

(iii) the first event happening in $(0, t)$ is that the intermediate cell with which we started at time, $t=0$ splits into two intermediate cells in the interval $(u, u+\Delta t) \subseteq(0, t)$;

(iv) the first event happening in $(0, t)$ is that the intermediate cell with which we started at time, $t=0$ splits into one intermediate cell and one malignant cell in the interval $(u, u+$ $\Delta t) \subseteq(0, t)$;

(v) the first event happening in $(0, t)$ is that the intermediate cell with which we started at time, $t=0$ splits into two malignant cells in the interval $(u, u+\Delta t) \subseteq(0, t)$;

(vi) the first event happening in $(0, t)$ is that the intermediate cell with which we started at time, $t=0$ dies (or differentiate) in the interval $(u, u+\Delta t) \subseteq(0, t)$.

Consequently, we have

$$
\begin{aligned}
\phi_{2}(t)=y e^{-b t}+ & \lambda_{21} \int_{0}^{t} e^{-b u} \phi_{1}(t-u) d u+\alpha_{22} \int_{0}^{t} e^{-b u}\left\{\phi_{2}(t-u)\right\}^{2} d u \\
& +z \alpha_{21} \int_{0}^{t} e^{-b u} \phi_{2}(t-u) d u+z^{2} \frac{\alpha_{20}}{b}\left(1-e^{-b t}\right)+\frac{\mu_{22}}{b}\left(1-e^{-b t}\right)
\end{aligned}
$$


International Journal of Mathematical, Engineering and Management Sciences

Vol. 5, No. 6, 1392-1408, 2020

https://doi.org/10.33889/IJMEMS.2020.5.6.103

where, $b=\lambda_{21}+\alpha_{22}+\alpha_{21}+\alpha_{20}+\mu_{22}$.

\section{Mean Numbers of Cells}

We consider the following conditional means:

$$
\begin{aligned}
& m_{X, j}^{(0)}(t)=\mathbb{E}[X(t) \mid X(0)=1, Y(0)=0, Z(0)=0, \eta(0)=j], j=1,2 ; \\
& m_{Y, j}^{(0)}(t)=\mathbb{E}[Y(t) \mid X(0)=1, Y(0)=0, Z(0)=0, \eta(0)=j], j=1,2 ; \\
& m_{Z, j}^{(0)}(t)=\mathbb{E}[Z(t) \mid X(0)=1, Y(0)=0, Z(0)=0, \eta(0)=j], j=1,2 ; \\
& m_{Y, j}^{(1)}(t)=\mathbb{E}[Y(t) \mid Y(0)=1, Z(0)=0, \eta(0)=j], j=1,2 ; \\
& m_{Z, j}^{(1)}(t)=\mathbb{E}[Z(t) \mid Y(0)=1, Z(0)=0, \eta(0)=j], j=1,2 .
\end{aligned}
$$

It is evident from the definitions of $\psi_{j}(t)$ and $\phi_{j}(t)$ that

$$
\begin{aligned}
& m_{X, j}^{(0)}(t)=\left[\frac{\partial \psi_{j}(t)}{\partial x}\right]_{x=1, y=1, z=1}, m_{Y, j}^{(0)}(t)=\left[\frac{\partial \psi_{j}(t)}{\partial y}\right]_{x=1, y=1, z=1}, \quad m_{Z, j}^{(0)}(t)=\left[\frac{\partial \psi_{j}(t)}{\partial z}\right]_{x=1, y=1, z=1} \\
& m_{Y, j}^{(1)}(t)=\left[\frac{\partial \phi_{j}(t)}{\partial y}\right]_{y=1, z=1}, m_{Z, j}^{(1)}(t)=\left[\frac{\partial \phi_{j}(t)}{\partial z}\right]_{y=1, z=1}, j=1,2 .
\end{aligned}
$$

Differentiating (1) and (2) partially with respect to $x$ and putting $x=1, y=1$ and $z=1$, we get

$$
\begin{gathered}
m_{X, 1}^{(0)}(t)=e^{-A t}+\lambda_{12} \int_{0}^{t} e^{-A u} m_{X, 2}^{(0)}(t-u) d u+2 L_{11} \int_{0}^{t} e^{-A u} m_{X, 1}^{(0)}(t-u) d u \\
m_{X, 2}^{(0)}(t)=e^{-B t}+\lambda_{21} \int_{0}^{t} e^{-B u} m_{X, 1}^{(0)}(t-u) d u+2 L_{22} \int_{0}^{t} e^{-B u} m_{X, 2}^{(0)}(t-u) d u \\
+L_{21} \int_{0}^{t} e^{-B u} m_{X, 2}^{(0)}(t-u) d u
\end{gathered}
$$

Differentiating (1) and (2) partially with respect to $y$ and putting $x=1, y=1$, and $z=1$, we get

$$
\begin{aligned}
& m_{Y, 1}^{(0)}(t)=\lambda_{12} \int_{0}^{t} e^{-A u} m_{Y, 2}^{(0)}(t-u) d u+2 L_{11} \int_{0}^{t} e^{-A u} m_{Y, 1}^{(0)}(t-u) d u \\
& m_{Y, 2}^{(0)}(t)=\lambda_{21} \int_{0}^{t} e^{-B u} m_{Y, 1}^{(0)}(t-u) d u+2 L_{22} \int_{0}^{t} e^{-B u} m_{Y, 2}^{(0)}(t-u) d u \\
& \quad+L_{21} \int_{0}^{t} e^{-B u}\left[m_{Y, 2}^{(0)}(t-u)+m_{Y, 2}^{(1)}(t-u)\right] d u+2 L_{20} \int_{0}^{t} e^{-B u} m_{Y, 2}^{(1)}(t-u) d u
\end{aligned}
$$


International Journal of Mathematical, Engineering and Management Sciences

Vol. 5, No. 6, 1392-1408, 2020

https://doi.org/10.33889/IJMEMS.2020.5.6.103

Differentiating (1) and (2) partially with respect to $z$ and putting $x=1, y=1$, and $z=1$, we get

$$
\begin{aligned}
m_{Z, 1}^{(0)}(t) & =\lambda_{12} \int_{0}^{t} e^{-A u} m_{Z, 2}^{(0)}(t-u) d u+2 L_{11} \int_{0}^{t} e^{-A u} m_{Z, 1}^{(0)}(t-u) d u \\
m_{Z, 2}^{(0)}(t) & =\lambda_{21} \int_{0}^{t} e^{-B u} m_{Z, 1}^{(0)}(t-u) d u+2 L_{22} \int_{0}^{t} e^{-B u} m_{Z, 2}^{(0)}(t-u) d u \\
& +L_{21} \int_{0}^{t} e^{-B u}\left[m_{Z, 2}^{(0)}(t-u)+m_{Z, 2}^{(1)}(t-u)\right] d u+2 L_{20} \int_{0}^{t} e^{-B u} m_{Z, 2}^{(1)}(t-u) d u .
\end{aligned}
$$

Differentiating (3) and (4) partially with respect to $y$ and, putting $y=1$ and $z=1$, we get

$$
\begin{gathered}
m_{Y, 1}^{(1)}(t)=e^{-a t}+\lambda_{12} \int_{0}^{t} e^{-a u} m_{Y, 2}^{(1)}(t-u) d u+2 \alpha_{11} \int_{0}^{t} e^{-a u} m_{Y, 1}^{(1)}(t-u) d u \\
m_{Y, 2}^{(1)}(t)=e^{-b t}+\lambda_{21} \int_{0}^{t} e^{-b u} m_{Y, 1}^{(1)}(t-u) d u+2 \alpha_{22} \int_{0}^{t} e^{-b u} m_{Y, 2}^{(1)}(t-u) d u \\
+\alpha_{21} \int_{0}^{t} e^{-b u} m_{Y, 2}^{(1)}(t-u) d u .
\end{gathered}
$$

Differentiating (3) and (4) partially with respect to $z$ and, putting $y=1$ and $z=1$, we get

$$
\begin{aligned}
& m_{Z, 1}^{(1)}(t)=\lambda_{12} \int_{0}^{t} e^{-a u} m_{Z, 2}^{(1)}(t-u) d u+2 \alpha_{11} \int_{0}^{t} e^{-a u} m_{Z, 1}^{(1)}(t-u) d u, \\
& m_{Z, 2}^{(1)}(t)=\lambda_{21} \int_{0}^{t} e^{-b u} m_{Z, 1}^{(1)}(t-u) d u+2 \alpha_{22} \int_{0}^{t} e^{-b u} m_{Z, 2}^{(1)}(t-u) d u \\
& \quad+\alpha_{21} \int_{0}^{t} e^{-b u} m_{Z, 2}^{(1)}(t-u) d u+\frac{\alpha_{21}}{b}\left(1-e^{-b t}\right)+2 \frac{\alpha_{20}}{b}\left(1-e^{-b t}\right) .
\end{aligned}
$$

Taking Laplace transform on both sides of (5) and (6), we get

$$
\begin{aligned}
& {\left[(s+A)-2 L_{11}\right] m_{X, 1}^{(0) *}(s)-\lambda_{12} m_{X, 2}^{(0) *}(s)=1} \\
& -\lambda_{21} m_{X, 1}^{(0) *}(s)+\left[(s+B)-2 L_{22}-L_{21}\right] m_{X, 2}^{(0) *}(s)=1 .
\end{aligned}
$$

Solving (15) and (16), we get,

$$
\begin{aligned}
& m_{X, 1}^{(0) *}(s)=\frac{s+\lambda_{12}+\lambda_{21}+p_{2}}{D(s)} \\
& m_{X, 2}^{(0) *}(s)=\frac{s+\lambda_{12}+\lambda_{21}-p_{1}}{D(s)}
\end{aligned}
$$


International Journal of Mathematical, Engineering and Management Sciences

Vol. 5, No. 6, 1392-1408, 2020

https://doi.org/10.33889/IJMEMS.2020.5.6.103

where,

$$
\begin{gathered}
p_{1}=L_{11}-D_{11}, p_{2}=L_{20}+D_{22}-L_{22} \\
D(s)=s^{2}+s\left(\lambda_{12}-p_{1}+\lambda_{21}+p_{2}\right)+\lambda_{12} p_{2}-\lambda_{21} p_{1}-p_{1} p_{2}
\end{gathered}
$$

The zeros of $D(s)$ are real and distinct, since the discriminant of $D(s)$ is given by

$$
\text { Discriminant }=\left[\left(\lambda_{12}-p_{1}\right)-\left(\lambda_{21}+p_{2}\right)\right]^{2}+4 \lambda_{12} \lambda_{21}>0 \text {. }
$$

Let $\theta_{1}$ and $\theta_{2}$ be the zeros of $D(s)$. Then, we have

$$
\begin{array}{cc}
\theta_{1}, \theta_{2}= & \frac{-\left(\lambda_{12}-p_{1}+\lambda_{21}+p_{2}\right) \pm \sqrt{\left[\left(\lambda_{12}-p_{1}\right)-\left(\lambda_{21}+p_{2}\right)\right]^{2}+4 \lambda_{12} \lambda_{21}}}{2}, \\
\theta_{1}+\theta_{2}=p_{1}-p_{2}-\lambda_{12}-\lambda_{21}, & \theta_{1} \theta_{2}=\lambda_{12} p_{2}-\lambda_{21} p_{1}-p_{1} p_{2}, \\
m_{X, 1}^{(0) *}(s)=\frac{s+p_{1}-\theta_{1}-\theta_{2}}{\left(s-\theta_{1}\right)\left(s-\theta_{2}\right)}, & m_{X, 2}^{(0) *}(s)=\frac{s-p_{2}-\theta_{1}-\theta_{2}}{\left(s-\theta_{1}\right)\left(s-\theta_{2}\right)} .
\end{array}
$$

Splitting into partial fractions, we obtain

$$
\begin{aligned}
& m_{X, 1}^{(0) *}(s)=\frac{1}{\left(\theta_{1}-\theta_{2}\right)}\left[\frac{p_{1}-\theta_{2}}{\left(s-\theta_{1}\right)}-\frac{p_{1}-\theta_{1}}{\left(s-\theta_{2}\right)}\right] \\
& m_{X, 2}^{(0) *}(s)=\frac{1}{\left(\theta_{1}-\theta_{2}\right)}\left[\frac{p_{2}+\theta_{1}}{\left(s-\theta_{2}\right)}-\frac{p_{2}+\theta_{2}}{\left(s-\theta_{1}\right)}\right]
\end{aligned}
$$

Taking inverse Laplace transform on both sides of (19) and (20), we get

$$
\begin{aligned}
& m_{X, 1}^{(0)}(t)=\frac{1}{\left(\theta_{1}-\theta_{2}\right)}\left[\left(p_{1}-\theta_{2}\right) e^{\theta_{1} t}-\left(p_{1}-\theta_{1}\right) e^{\theta_{2} t}\right] \\
& m_{X, 2}^{(0)}(t)=\frac{1}{\left(\theta_{1}-\theta_{2}\right)}\left[\left(p_{2}+\theta_{1}\right) e^{\theta_{2} t}-\left(p_{2}+\theta_{2}\right) e^{\theta_{1} t}\right]
\end{aligned}
$$

To compute $m_{Y, 1}^{(0)}(t)$ and $m_{Z, 1}^{(0)}(t)$, we require $m_{Y, 1}^{(1)}(t), m_{Y, 2}^{(1)}(t), m_{Z, 1}^{(1)}(t)$, and $m_{Z, 2}^{(1)}(t)$. To obtain $m_{Y, 1}^{(1)}(t)$, and $m_{Y, 2}^{(1)}(t)$, we take Laplace transform on both sides of (11) and (12), and get

$$
\begin{aligned}
& \left(s+a-2 \alpha_{11}\right) m_{Y, 1}^{(1) *}(s)-\lambda_{12} m_{Y, 2}^{(1) *}(s)=1 \\
& -\lambda_{21} m_{Y, 1}^{(1) *}(s)+\left(s+b-2 \alpha_{22}-\alpha_{21}\right) m_{Y, 2}^{(1) *}(s)=1
\end{aligned}
$$

Solving (23) and (24), we get

$$
m_{Y, 1}^{(1) *}(s)=\frac{s+\lambda_{12}+\lambda_{21}+\alpha_{20}+\mu_{22}-\alpha_{22}}{\Delta(s)}
$$


International Journal of Mathematical, Engineering and Management Sciences

Vol. 5, No. 6, 1392-1408, 2020

https://doi.org/10.33889/IJMEMS.2020.5.6.103

$m_{Y, 2}^{(1) *}(s)=\frac{s+\lambda_{12}+\lambda_{21}+\mu_{11}-\alpha_{11}}{\Delta(s)}$

where,

$$
\begin{aligned}
q_{1} & =\alpha_{11}-\mu_{11}, q_{2}=\alpha_{20}+\mu_{22}-\alpha_{22}, \\
\Delta(s) & =s^{2}+\left(\lambda_{12}+\lambda_{21}-q_{1}+q_{2}\right)+\left(\lambda_{12}-q_{1}\right)\left(\lambda_{21}+q_{2}\right)-\lambda_{12} \lambda_{21} .
\end{aligned}
$$

The zeros of $\Delta(s)$ are real and distinct, since the discriminant of $\Delta(s)$ is given by

Discriminant $=\left[\left(\lambda_{12}-q_{1}\right)-\left(\lambda_{21}+q_{2}\right)\right]^{2}+4 \lambda_{12} \lambda_{21}>0$.

Let $\omega_{1}$ and $\omega_{2}$ be the zeros of $\Delta(s)$. Then, we have

$$
\begin{gathered}
\omega_{1}, \omega_{2}=\frac{-\left(\lambda_{12}-q_{1}+\lambda_{21}+q_{2}\right) \pm \sqrt{\left[\left(\lambda_{12}-q_{1}\right)-\left(\lambda_{21}+q_{2}\right)\right]^{2}+4 \lambda_{12} \lambda_{21}}}{2}, \\
\omega_{1}+\omega_{2}=q_{1}-q_{2}-\lambda_{12}-\lambda_{21}, \quad \omega_{1} \omega_{2}=\lambda_{12} q_{2}-\lambda_{21} q_{1}-q_{1} q_{2}, \\
m_{Y, 1}^{(1) *}(s)=\frac{s+q_{1}-\omega_{1}-\omega_{2}}{\left(s-\omega_{1}\right)\left(s-\omega_{2}\right)}, \quad m_{Y, 2}^{(1) *}(s)=\frac{s-q_{2}-\omega_{1}-\omega_{2}}{\left(s-\omega_{1}\right)\left(s-\omega_{2}\right)} .
\end{gathered}
$$

Splitting into partial fractions, (25) and (26) yield

$$
\begin{aligned}
& m_{Y, 1}^{(1) *}(s)=\frac{1}{\left(\omega_{1}-\omega_{2}\right)}\left[\frac{q_{1}-\omega_{2}}{\left(s-\omega_{1}\right)}-\frac{q_{1}-\omega_{1}}{\left(s-\omega_{2}\right)}\right] \\
& m_{Y, 2}^{(1) *}(s)=\frac{1}{\left(\omega_{1}-\omega_{2}\right)}\left[\frac{q_{2}+\omega_{1}}{\left(s-\omega_{2}\right)}-\frac{q_{2}+\omega_{2}}{\left(s-\omega_{1}\right)}\right]
\end{aligned}
$$

Taking inverse Laplace transform on both sides of (27) and (28), we get

$$
\begin{aligned}
& m_{Y, 1}^{(1)}(t)=\frac{1}{\left(\omega_{1}-\omega_{2}\right)}\left[\left(q_{1}-\omega_{2}\right) e^{\omega_{1} t}-\left(q_{1}-\omega_{1}\right) e^{\omega_{2} t}\right] \\
& m_{Y, 2}^{(1)}(t)=\frac{1}{\left(\omega_{1}-\omega_{2}\right)}\left[\left(q_{2}+\omega_{1}\right) e^{\omega_{2} t}-\left(q_{2}+\omega_{2}\right) e^{\omega_{1} t}\right]
\end{aligned}
$$

Next, to find $m_{Z, 1}^{(1)}(t)$ and $m_{Z, 2}^{(1)}(t)$, we take Laplace transform on both sides of (13) and (14) and obtain the following equations:

$$
\begin{aligned}
& \left(s+a-2 \alpha_{11}\right) m_{Z, 1}^{(1) *}(s)-\lambda_{12} m_{Z, 2}^{(1) *}(s)=0 \\
& -\lambda_{21} m_{Z, 1}^{(1) *}(s)+\left(s+b-2 \alpha_{22}-\alpha_{21}\right) m_{Z, 2}^{(1) *}(s)==\frac{\alpha_{21}+2 \alpha_{20}}{s}
\end{aligned}
$$


International Journal of Mathematical, Engineering and Management Sciences

Vol. 5, No. 6, 1392-1408, 2020

https://doi.org/10.33889/IJMEMS.2020.5.6.103

Solving (31) and (32), we obtain

$$
\begin{aligned}
& m_{Z, 1}^{(1) *}(s)=\frac{\lambda_{12}\left(\alpha_{21}+2 \alpha_{20}\right)}{s\left(s-\omega_{1}\right)\left(s-\omega_{2}\right)} \\
& m_{Z, 2}^{(1) *}(s)=\frac{\left(\alpha_{21}+2 \alpha_{20}\right)\left(s+\lambda_{12}+\mu_{11}-\alpha_{11}\right)}{s\left(s-\omega_{1}\right)\left(s-\omega_{2}\right)}
\end{aligned}
$$

Taking inverse Laplace transform on both sides of (33) and (34), we obtain

$$
\begin{aligned}
& m_{Z, 1}^{(1)}(t)=\frac{\lambda_{12}\left(\alpha_{21}+2 \alpha_{20}\right)}{\omega_{1} \omega_{2}}\left[1+\frac{1}{\omega_{1}-\omega_{2}}\left\{\omega_{2} e^{\omega_{1} t}-\omega_{1} e^{\omega_{2} t}\right\}\right] \\
& m_{Z, 2}^{(1)}(t)=\frac{\alpha_{21}+2 \alpha_{20}}{\omega_{1} \omega_{2}}\left[\left(\lambda_{12}-q_{1}\right)+\frac{\omega_{2}\left(\omega_{1}+\lambda_{12}-q_{1}\right) e^{\omega_{1} t}}{\left(\omega_{1}-\omega_{2}\right)}-\frac{\omega_{1}\left(\omega_{2}+\lambda_{12}-q_{1}\right) e^{\omega_{2} t}}{\left(\omega_{1}-\omega_{2}\right)}\right]
\end{aligned}
$$

Now, we are in a position to find $m_{Y, 1}^{(0)}(t), m_{Y, 2}^{(0)}(t), m_{Z, 1}^{(0)}(t)$ and $m_{Z, 2}^{(0)}(t)$. Taking Laplace transform on both sides of (7) and (8), we get

$$
\begin{aligned}
& \left(s+A-2 L_{11}\right) m_{Y, 1}^{(0) *}(s)-\lambda_{12} m_{Y, 2}^{(0) *}(s)=0 \\
& -\lambda_{21} m_{Y, 1}^{(0) *}(s)+\left(s+B-2 L_{22}-L_{21}\right) m_{Y, 2}^{(0) *}(s)=\left(L_{21}+2 L_{20}\right) m_{Y, 2}^{(1) *}(s)
\end{aligned}
$$

Solving (37) and (38), we get

$$
\begin{aligned}
& m_{Y, 1}^{(0) *}(s)=\frac{\lambda_{12}\left(L_{21}+2 L_{20}\right)}{\left(s-\theta_{1}\right)\left(s-\theta_{2}\right)} m_{Y, 2}^{(1) *}(s) \\
& m_{Y, 2}^{(0) *}(s)=\frac{\left(L_{21}+2 L_{20}\right)\left(s+\lambda_{12}-p_{1}\right)}{\left(s-\theta_{1}\right)\left(s-\theta_{2}\right)} m_{Y, 2}^{(1) *}(s)
\end{aligned}
$$

Taking inverse Laplace transform on both sides of (39) and (40), we get

$$
\begin{gathered}
m_{Y, 1}^{(0)}(t)=\frac{\lambda_{12}\left(L_{21}+2 L_{20}\right)}{\left(\theta_{1}-\theta_{2}\right)\left(\omega_{1}-\omega_{2}\right)}\left[\left(q_{2}+\omega_{1}\right)\left\{\frac{e^{\theta_{1} t}-e^{\omega_{2} t}}{\theta_{1}-\omega_{2}}-\frac{e^{\theta_{2} t}-e^{\omega_{2} t}}{\theta_{2}-\omega_{2}}\right\}\right. \\
\left.-\left(q_{2}+\omega_{2}\right)\left\{\frac{e^{\theta_{1} t}-e^{\omega_{1} t}}{\theta_{1}-\omega_{1}}-\frac{e^{\theta_{2} t}-e^{\omega_{1} t}}{\theta_{2}-\omega_{1}}\right\}\right] \\
m_{Y, 2}^{(0)}(t)=\frac{\left(L_{21}+2 L_{20}\right)}{\left(\theta_{1}-\theta_{2}\right)\left(\omega_{1}-\omega_{2}\right)}\left[( q _ { 2 } + \omega _ { 1 } ) \left\{\left(\theta_{1}+\lambda_{12}-p_{1}\right)\left(\frac{e^{\theta_{1} t}-e^{\omega_{2} t}}{\theta_{1}-\omega_{2}}\right)\right.\right. \\
\left.-\left(\theta_{2}+\lambda_{12}-p_{1}\right)\left(\frac{e^{\theta_{2} t}-e^{\omega_{2} t}}{\theta_{2}-\omega_{2}}\right)\right\}-\left(q_{2}+\omega_{2}\right)\left\{\left(\theta_{1}+\lambda_{12}-p_{1}\right)\left(\frac{e^{\theta_{1} t}-e^{\omega_{1} t}}{\theta_{1}-\omega_{1}}\right)\right.
\end{gathered}
$$


International Journal of Mathematical, Engineering and Management Sciences

Vol. 5, No. 6, 1392-1408, 2020

https://doi.org/10.33889/IJMEMS.2020.5.6.103

$$
\left.\left.-\left(\theta_{2}+\lambda_{12}-p_{1}\right)\left(\frac{e^{\theta_{2} t}-e^{\omega_{1} t}}{\theta_{2}-\omega_{1}}\right)\right\}\right]
$$

Replacing $Y$ by $Z$ in (7) and (8), we get (9) and (10). Consequently, the expressions for $m_{Z, 1}^{(0)}(t)$ and $m_{Z, 2}^{(0)}(t)$ are obtained along the similar lines of (41) and (42) and we have

$$
\begin{gathered}
m_{Z, 1}^{(0)}(t)=\frac{\lambda_{12}\left(L_{21}+2 L_{20}\right)\left(\alpha_{21}+2 \alpha_{20}\right)}{\omega_{1} \omega_{2}\left(\theta_{1}-\theta_{2}\right)}\left[\left(\lambda_{12}-q_{1}\right)\left\{\left(\frac{e^{\theta_{1} t}-1}{\theta_{1}}\right)-\left(\frac{e^{\theta_{2} t}-1}{\theta_{2}}\right)\right\}\right. \\
+\frac{\omega_{2}\left(\omega_{1}+\lambda_{12}-q_{1}\right)}{\omega_{1}-\omega_{2}}\left\{\left(\frac{e^{\theta_{1} t}-e^{\omega_{1} t}}{\theta_{1}-\omega_{1}}\right)-\left(\frac{e^{\theta_{2} t}-e^{\omega_{1} t}}{\theta_{2}-\omega_{1}}\right)\right\} \\
\left.-\frac{\omega_{1}\left(\omega_{2}+\lambda_{12}-q_{1}\right)}{\omega_{1}-\omega_{2}}\left\{\left(\frac{e^{\theta_{1} t}-e^{\omega_{2} t}}{\theta_{1}-\omega_{2}}\right)-\left(\frac{e^{\theta_{2} t}-e^{\omega_{2} t}}{\theta_{2}-\omega_{2}}\right)\right\}\right] \\
m_{z, 2}^{(0)}(t)=\frac{\left(L_{21}+2 L_{20}\right)\left(\alpha_{21}+2 \alpha_{20}\right)}{\omega_{1} \omega_{2}\left(\theta_{1}-\theta_{2}\right)}\left[( \lambda _ { 1 2 } - q _ { 1 } ) \left\{\left(\theta_{1}+\lambda_{12}-p_{1}\right)\left(\frac{e^{\theta_{1} t}-1}{\theta_{1}}\right)\right.\right. \\
+\frac{\omega_{2}\left(\omega_{1}+\lambda_{12}-q_{1}\right)}{\left(\omega_{1}-\omega_{2}\right)}\left\{\left(\theta_{1}+\lambda_{12}-p_{1}\right)\left(\frac{e^{\theta_{2} t}-1}{\theta_{2}}\right)\right\} \\
\left.\frac{\omega_{1}\left(\omega_{2}+\lambda_{12}-q_{1}\right)}{\left(\omega_{1}-\omega_{2}\right)}\left\{\left(\theta_{1}+\lambda_{12}-p_{1}\right)\left(\frac{\left.e^{\theta_{1} t}-e^{\omega_{2} t}\right)}{\left(\theta_{1}-\omega_{2}\right)}\right)-\left(\theta_{2}+\lambda_{12}-p_{1}\right)\left(\frac{e^{\theta_{2} t}-e^{\omega_{2} t}}{\left(\theta_{2}-\omega_{2}\right)}\right)\right\}\right]
\end{gathered}
$$

Equations 21, 22, 29, 30, 35,36, 41, 42, 43 and 44 provide explicitly the mean values of the number of normal, intermediate, and malignant cells which are present at time $t$. If we fix the threshold value for the emergence of a malignant cell as 1 , then, by solving the equation $v_{1} m_{z, 1}^{(0)}(t)+$ $v_{2} m_{Z, 2}^{(0)}(t)=1$ for $t$, we can have a rough estimate of the time of occurrence of a malignant cell in the population.

\section{A Numerical Illustration}

For the purpose of illustration, we fix the parameters as follows:

$$
\begin{aligned}
& \lambda_{12}=0.6 ; \lambda_{21}=0.3 ; L_{11}=0.7 ; L_{20}=0.6 ; L_{21}=0.7 ; L_{22}=0.8 ; \quad D_{11}=0.2 ; D_{22}=0.4 ; \\
& \alpha_{11}=0.7 ; \alpha_{20}=0.5 ; \alpha_{21}=0.6 ; \alpha_{22}=0.7 ; \quad \mu_{11}=0.3 ; \mu_{22}=0.2 .
\end{aligned}
$$

\subsection{Two Mutation Model}

We have computed the mean number of normal cells in the case of two mutation model for times varying from 0.1 to 1.5 in both levels of the environment and depicted the growth in Figure 2. In Table 1, we have exhibited a comparative picture for the growth of the normal cells in both levels of the environment. It is seen from Table 1 as well as from Figure 2 that the growth is inhibited in level 2. We also find from Table 1 that when the population is initiated with 1 normal cell in level 1 of the environment at time $t=0$, the population of normal cells shows an increasing trend right from the time $t=0$. On the other hand, when the population is initiated with 1 normal cell in level 2 of the environment at time $t=0$, the population of normal cells shows a decreasing trend in the 
International Journal of Mathematical, Engineering and Management Sciences

Vol. 5, No. 6, 1392-1408, 2020

https://doi.org/10.33889/IJMEMS.2020.5.6.103

beginning and then an increasing trend right from the time $t=1.4$ onwards. This is so because the rates in environment level 1 are higher than those in level 2.

Table 1. Two mutation: comparison of growth of normal cell

\begin{tabular}{|c|c|c|c|c|c|c|c|c|}
\hline$t$ & $m_{X, 1}^{(0)}(t)$ & $m_{X, 2}^{(0)}(t)$ & $t$ & $m_{X, 1}^{(0)}(t)$ & $m_{X, 2}^{(0)}(t)$ & $t$ & $m_{X, 1}^{(0)}(t)$ & $m_{X, 2}^{(0)}(t)$ \\
\hline 0.1 & 1.0492 & 0.98122 & 0.6 & 1.2748 & 0.91935 & 1.1 & 1.4779 & 0.89939 \\
\hline 0.2 & 1.0968 & 0.96477 & 0.7 & 1.3168 & 0.91243 & 1.2 & 1.5168 & 0.89935 \\
\hline 0.3 & 1.1431 & 0.9505 & 0.8 & 1.358 & 0.90708 & 1.3 & 1.5555 & 0.90044 \\
\hline 0.4 & 1.1881 & 0.93826 & 0.9 & 1.3985 & 0.90318 & 1.4 & 1.5938 & 0.90261 \\
\hline 0.5 & 1.2319 & 0.92792 & 1.0 & 1.4384 & 0.90064 & 1.5 & 1.6319 & 0.90579 \\
\hline
\end{tabular}

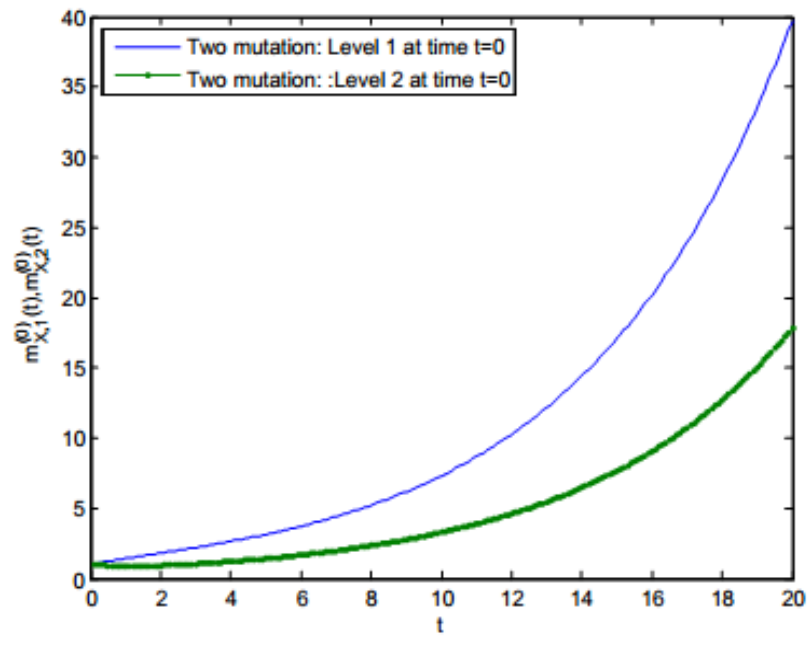

Figure 2. Graphs of $m_{X, j}^{(0)}(t), j=1,2$

Next, we computed the mean number of intermediate cells in the case of two mutation model for times varying from 0.1 to 1.5 in both levels of the environment and depicted the growth in Figure 3 . In Table 2, we have exhibited a comparative picture for the growth of the intermediate cells in both levels of the environment. It is seen from Table 2 as well as from Figure 3 that the growth rate of intermediate cells is slightly more when we start with environment in level 1 than the growth rate of intermediate cells when we start with environment in level 2; but, after time point 3.3, this growth rate is lowered for the case in which the environment begins in level 2 . This is so because the rates in environment 1 are greater than those in level 2.

Table 2. Two mutation: comparison of growth of intermediate cells

\begin{tabular}{|c|c|c|c|c|c|c|c|c|}
\hline$t$ & $m_{Y, 1}^{(0)}(t)$ & $m_{Y, 2}^{(0)}(t)$ & $t$ & $m_{Y, 1}^{(0)}(t)$ & $m_{Y, 2}^{(0)}(t)$ & $t$ & $m_{Y, 1}^{(0)}(t)$ & $m_{Y, 2}^{(0)}(t)$ \\
\hline 0.5 & 0.12943 & 0.84456 & 3.0 & 3.2114 & 3.4871 & 3.5 & 4.1391 & 3.9212 \\
\hline 1.0 & 0.47378 & 1.5241 & 3.1 & 3.3902 & 3.5738 & 4.0 & 5.1496 & 4.3636 \\
\hline 1.5 & 0.98277 & 2.0932 & 3.2 & 3.5724 & 3.6604 & 4.5 & 6.2428 & 4.8241 \\
\hline 2.0 & 1.6222 & 2.5919 & 3.3 & 3.758 & 3.7471 & 5.0 & 7.421 & 5.3102 \\
\hline 2.5 & 2.3698 & 3.0494 & 3.4 & 3.9469 & 3.834 & 5.5 & 8.6885 & 5.8285 \\
\hline
\end{tabular}


International Journal of Mathematical, Engineering and Management Sciences

Vol. 5, No. 6, 1392-1408, 2020

https://doi.org/10.33889/IJMEMS.2020.5.6.103

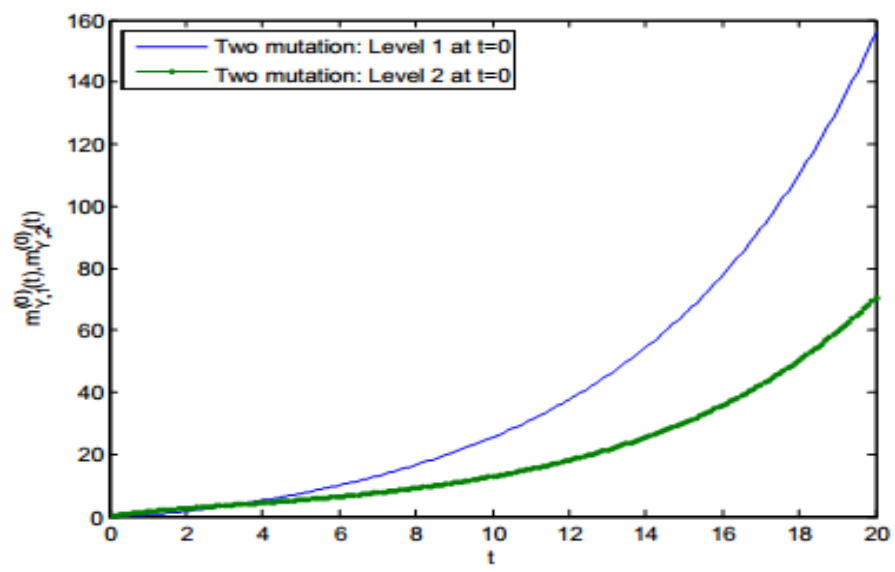

Figure 3. Graphs of $m_{Y, j}^{(0)}(t), j=1,2$

In Table 3, we have exhibited a comparative picture for the growth of the malignant cells in both levels of the environment. It is seen from Table 3 as well as from Figure 4 that the growth rate of intermediate cells is slightly more when we start with environment in level 1 than the growth rate of intermediate cells when we start with environment in level 2; but, after time point 3.3, this growth rate is lowered for the case in which the environment begins in level 2 . This is so because the rates in environment 1 are greater than those in level 2.

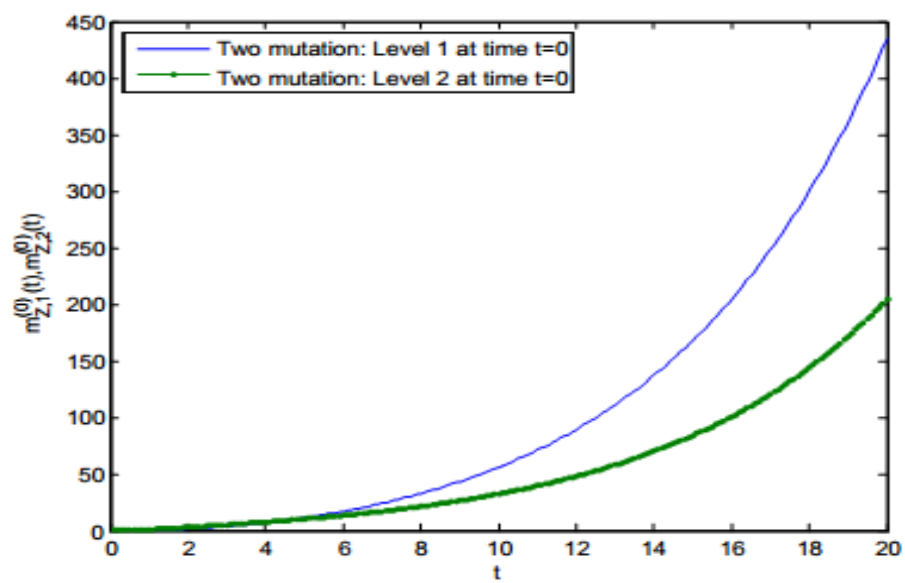

Figure 4. Graphs of $m_{Z, j}^{(0)}(t), j=1,2$

Table 3. Two mutation: comparison of growth of malignant cells

\begin{tabular}{|c|c|c|c|c|c|c|c|c|}
\hline$t$ & $m_{Z, 1}^{(0)}(t)$ & $m_{Z, 2}^{(0)}(t)$ & $t$ & $m_{Z, 1}^{(0)}(t)$ & $m_{Z, 2}^{(0)}(t)$ & $t$ & $m_{Z, 1}^{(0)}(t)$ & $m_{Z, 2}^{(0)}(t)$ \\
\hline 0.5 & 0.032089 & 0.30879 & 3.0 & 3.5234 & 5.1745 & 4.4 & 8.4743 & 8.6361 \\
\hline 1.0 & 0.21934 & 1.0225 & 3.5 & 5.0385 & 6.3541 & 4.5 & 8.9136 & 8.905 \\
\hline 1.5 & 0.63979 & 1.9401 & 4.0 & 6.8333 & 7.5932 & 4.6 & 9.3647 & 9.1773 \\
\hline 2.0 & 1.3252 & 2.9619 & 4.2 & 7.6308 & 8.1084 & 5.0 & 11.2888 & 10.3039 \\
\hline 2.5 & 2.2858 & 4.0445 & 4.3 & 8.0467 & 8.3706 & 5.5 & 13.9722 & 11.8049 \\
\hline
\end{tabular}


International Journal of Mathematical, Engineering and Management Sciences

Vol. 5, No. 6, 1392-1408, 2020

https://doi.org/10.33889/IJMEMS.2020.5.6.103

\subsection{One Mutation Model}

We have computed the mean number of normal cells and malignant cells in the case of one mutation model for times varying from 0.1 to 1.5 in both levels of the environment and depicted the growth in Figure 5 and Figure 6.

Table 4. One mutation: comparison of growth of normal cells

\begin{tabular}{|c|c|c|c|c|c|c|c|c|}
\hline$t$ & $m_{Y, 1}^{(1)}(t)$ & $m_{Y, 2}^{(1)}(t)$ & $t$ & $m_{Y, 1}^{(1)}(t)$ & $m_{Y, 2}^{(1)}(t)$ & $t$ & $m_{Y, 1}^{(1)}(t)$ & $m_{Y, 2}^{(1)}(t)$ \\
\hline 0.1 & 1.0377 & 0.99967 & 1.8 & 1.2583 & 0.93587 & 4.4 & 1.1183 & 0.78633 \\
\hline 0.3 & 1.1008 & 0.99725 & 1.9 & 1.2577 & 0.93038 & 4.6 & 1.1035 & 0.77519 \\
\hline 0.5 & 1.1498 & 0.99282 & 2.0 & 1.2563 & 0.92483 & 4.8 & 1.0888 & 0.76417 \\
\hline 0.7 & 1.187 & 0.98677 & 2.4 & 1.2446 & 0.90203 & 5.0 & 1.0741 & 0.75327 \\
\hline 0.9 & 1.2146 & 0.97939 & 2.5 & 1.2404 & 0.89624 & 5.2 & 1.0594 & 0.74251 \\
\hline 1.1 & 1.2343 & 0.97096 & 3.0 & 1.2143 & 0.86702 & 5.4 & 1.0447 & 0.73189 \\
\hline 1.3 & 1.2473 & 0.96169 & 3.2 & 1.2021 & 0.8553 & 5.6 & 1.0302 & 0.7214 \\
\hline 1.5 & 1.255 & 0.95174 & 3.6 & 1.1756 & 0.83199 & 5.8 & 1.0158 & 0.71104 \\
\hline 1.6 & 1.257 & 0.94656 & 4.0 & 1.1474 & 0.80896 & 5.9 & 1.0086 & 0.70592 \\
\hline 1.7 & 1.2581 & 0.94126 & 4.2 & 1.1329 & 0.79759 & 6.0 & 1.0015 & 0.70083 \\
\hline
\end{tabular}

In Table 4, we have exhibited a comparative picture for the growth of normal cells in both levels of the environment. It is seen from Table 4 as well as from Figure 5 that the growth rate of normal cells is always more when we start with environment in level 1 than the growth rate of normal cells when we start with environment in level 2 . This is so because the rates in environment 1 are higher than those in level 2.

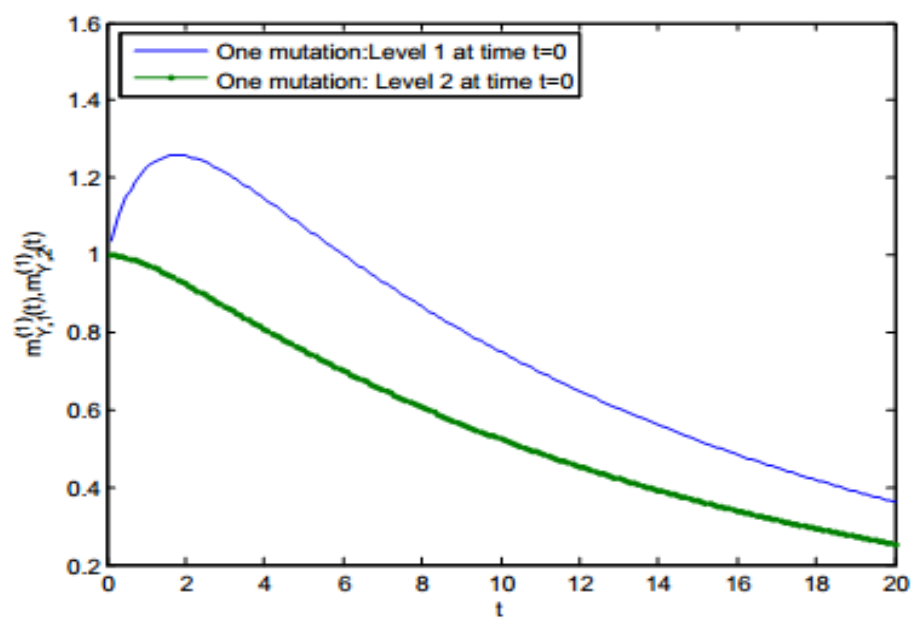

Figure 5. Graphs of $m_{Y, j}^{(1)}(t), j=1,2$

In Table 5, we have exhibited a comparative picture for the growth of malignant cells in both levels of the environment. It is seen from Table 5 as well as from Figure 6 that the growth rate of malignant cells is slightly higher up to time point 3.2 when we start with environment in level 1 at time $t=0$ than the growth rate of malignant cells when we start with environment in level 2 at time $t=0$. After the time point 3.3, this growth rate is lowered for the case in which the environment begins in level 2. This is so because the rates in environment 1 are higher than those in level 2. 
International Journal of Mathematical, Engineering and Management Sciences

Vol. 5, No. 6, 1392-1408, 2020

https://doi.org/10.33889/IJMEMS.2020.5.6.103

Table 5. One mutation: comparison of growth of malignant cells

\begin{tabular}{|c|c|c|c|c|c|c|c|c|}
\hline$t$ & $m_{Z, 1}^{(1)}(t)$ & $m_{Z, 2}^{(1)}(t)$ & $t$ & $m_{Z, 1}^{(1)}(t)$ & $m_{Z, 2}^{(1)}(t)$ & $t$ & $m_{Z, 1}^{(1)}(t)$ & $m_{Z, 2}^{(1)}(t)$ \\
\hline 0.1 & 0.0046437 & 0.15379 & 2.3 & 1.3113 & 1.7991 & 4.5 & 3.1183 & 2.3601 \\
\hline 0.3 & 0.039166 & 0.42733 & 2.5 & 1.4757 & 1.8686 & 4.7 & 3.2759 & 2.398 \\
\hline 0.5 & 0.10213 & 0.66183 & 2.7 & 1.6415 & 1.9325 & 4.9 & 3.4318 & 2.4348 \\
\hline 0.7 & 0.18823 & 0.86381 & 2.9 & 1.808 & 1.9916 & 5.1 & 3.5857 & 2.4706 \\
\hline 0.9 & 0.29305 & 1.0387 & 3.1 & 1.9747 & 2.0468 & 5.3 & 3.7378 & 2.5054 \\
\hline 1.1 & 0.41296 & 1.1909 & 3.3 & 2.1411 & 2.0986 & 5.5 & 3.888 & 2.5394 \\
\hline 1.3 & 0.54491 & 1.3242 & 3.5 & 2.3069 & 2.1475 & 5.7 & 4.0362 & 2.5726 \\
\hline 1.5 & 0.68641 & 1.4417 & 3.7 & 2.4719 & 2.1938 & 5.9 & 4.1825 & 2.6051 \\
\hline 1.7 & 0.83537 & 1.5459 & 3.9 & 2.6356 & 2.238 & 6.0 & 4.2549 & 2.621 \\
\hline 1.9 & 0.99009 & 1.6391 & 4.1 & 2.7981 & 2.2803 & 6.1 & 4.3268 & 2.6369 \\
\hline 2.1 & 1.1491 & 1.723 & 4.3 & 2.959 & 2.3209 & 6.2 & 4.3983 & 2.6526 \\
\hline
\end{tabular}

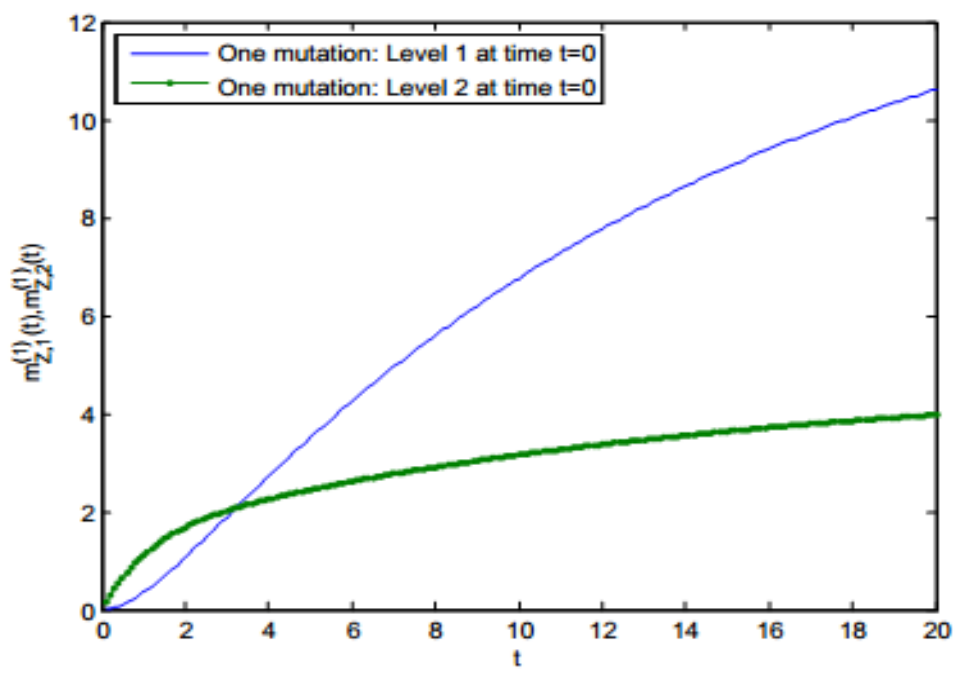

Figure 6. Graph of $m_{Z, j}^{(1)}(t), j=1,2$

\section{Conclusion}

In the present paper, we studied a two-stage mutation stochastic model of carcinogenesis in a two level random environment. For the values of the growth parameters already available in the earlier papers, we computed the mean values of the number of normal cells, intermediate cells and malignant cells in the population at any time $t$. We found that the appearance of malignant cells is delayed in the two-stage model in comparison with the one-mutation model. Further, we also found that the appearance of malignant cells is quicker in environment level 2 than in environment level 1. This observation justifies the fact that environment has a definite influence in carcinogenesis. In a future research, an attempt would be made to include more levels in the environment in order to highlight the influence of different levels of environment on the mutational behavior of normal and intermediate cells and compare the changes in the production of malignant cells with the results obtained in the present paper. 
International Journal of Mathematical, Engineering and Management Sciences

Vol. 5, No. 6, 1392-1408, 2020

https://doi.org/10.33889/IJMEMS.2020.5.6.103

\section{Conflict of Interest}

The authors have equal contribution in this work and it is declared that there is no conflict of interest for this publication.

\section{Acknowledgment}

The authors express their sincere gratitude to the referees for their valuable suggestions towards the improvement of the paper. One of the authors V.S.S. Yadavalli is thankful to NRF South Africa for their financial support.

\section{References}

Asmussen, S., \& Hering, H. (1983). Branching processes. Boston, Birkhäuser.

Athreya, K.B., \& Ney, P.E. (1972). Branching processes, Springer-Verlag, Berlin.

Bellman, R., Kalaba, R., \& Wing, G.M. (1960). Invariant imbedding and mathematical physics. I. particle processes. Journal of Mathematical Physics, 1(4), 280-308.

Harris, T.E. (1963). The theory of branching processes. Springer-Verlag, Berlin.

Hochberg, M.E., \& Noble, R.J. (2017). A framework for how environment contributes to cancer risk. Ecology Letters, 20(2), 117-134.

Martincorena, I., Raine, K.M., Gerstung, M., Dawson, K.J., Haase, K., Loo, P.V., Davies, H., Stratton, M.R., \& Campbell, P.J. (2017). Universal patterns of selection in cancer and somatic tissues. Cell, 171,1-13.

Mode, C.J. (1971). Multitype branching processes: theory and applications (Vol. 34). American Elsevier, New York.

Moolgavkar, S.H., \& Venzon, D.J. (1979). Two-event models for carcinogenesis: incidence curves for childhood and adult tumors. Mathematical Biosciences, 47(1-2), 55-77.

Reddy, B.Y., Miller, D.M., \& Tsao, H. (2017). Somatic driver mutations in melanoma. Cancer, 123(S11), 2104-2117.

Rozhok, A., \& DeGregori, J. (2019). A generalized theory of age-dependent carcinogenesis. ELife. 2019;8:e39950 DOI: 10.7554/eLife.39950.

Rozhok, A.I., Salstrom, J.L., \& DeGregori, J. (2016). Stochastic modeling reveals an evolutionary mechanism underlying elevated rates of childhood leukemia. Proceedings of the National Academy of Sciences, 113(4), 1050-1055.

Srinivasan, S.K. (1969). Stochastic theory and cascade processes, American Elsevier, New York.

Sun, S., Klebaner, F., \& Tian, T. (2014). A new model of time scheme for progression of colorectal cancer. BMC Systems Biology, 8, S2. https://doi.org/10.1186/1752-0509-8-S3-S2.

Tomasetti, C., \& Vogelstein, B. (2015). Variation in cancer risk among tissues can be explained by the number of stem cell divisions. Science, 347(6217), 78-81.

Wolf, D.C., Cohen, S.M., Boobis, A.R., Dellarco, V.L., Fenner-Crisp, P.A., Moretto, A., Pastoor, T.P., Schoeny, R.S., Seed, J.G., \& Doe, J.E. (2019). Chemical carcinogenicity revisited 1: a unified theory of carcinogenicity based on contemporary knowledge. Regulatory Toxicology and Pharmacology, 103, $86-92$. 